\title{
Operating Room Scheduling Data Base Analysis for Scheduling
}

\author{
Walton M. Hancock, Paul F. Walter, Roy A. More, and \\ Noah D. Glick
}

\begin{abstract}
A methodology to determine and maintain the schedule times used in a computer-based OR Scheduling System is presented. State-of-the-art equations to be used for different scheduling situations are presented and discussed with the attendant data base reduction methodology to provide the scheduling parameters. An example is given of the data reduction methodology as well as suggestions for maintenance of the data base.
\end{abstract}

\section{INTRODUCTION}

As the use of microcomputers becomes more extensive in the scheduling of patients in operating rooms (OR), great potential exists to improve the scheduling process so that many of the present administrative problems, such as schedule slippage, low utilization, and excessive overtime can be reduced.

This paper discusses a very important aspect of the use of microcomputers in the OR to determine and maintain schedule times that are as accurate as possible. Typical OR scheduling systems usually are based on the average time $(\bar{X})$ for the procedure(s) with the values based on experience or statistical analysis. If these times are assumed to be normally distributed and are used to schedule the OR procedures where everyone is expected to be ready to begin at the scheduled time, then the probability will be approximately 0.50 that the procedure will be finished within the allotted period; thus, the probability of being able to start the second procedure on time is 0.50 . Likewise, the probability of starting the third procedure on time is between 0.25 and 0.50 .

This methodology can result in poor utilization because surgeons, especially the busier ones, will want to schedule cases only where they have a reasonable probability of

From the Industrial \& Operations Engineering Department, The University of Michigan, Ann Arbor, Michigan 48109-2110; the Walter Software Engineering, Whitmore Lake, Michigan 48189; the Health Management Advisors, Ann Arbor, Michigan 48103; and the Health Services Research \& Development, Veterans Administration, Ann Arbor, Michigan 48106. 
starting on time, namely, the AM cases, or when they can follow themselves. Also, poor utilization can result because OR personnel are reluctant to schedule cases in the afternoon due to the difficulty in determining a schedule permitting them to finish before the end of the shift.

If more appropriate scheduling times could be provided and used as part of the scheduling process, then the administrative problems described above could be greatly reduced. Procedures could be scheduled throughout the day with much higher probabilities that they would start on time. ${ }^{1,2}$ In addition, OR staff could be forewarned with greater precision when overtime would be required.

\section{PROCEDURE TIME COMPONENT EQUATIONS}

If the distributions of time required for an OR procedure were normally distributed, Equation (1) could be used accurately to predict the amount of time required for the case at whatever level of confidence the user desired.

$$
P_{\mathrm{ij}} \cdots_{\mathrm{m}}=\mathrm{X}_{\mathrm{ij}} \cdots \mathrm{m}+Z * S_{\mathrm{ij}} \cdots \mathrm{m},
$$

where $P$ is the time to be allotted for the procedure. $\mathrm{ij} \ldots \mathrm{m}$ are subscripts indicating surgeon, case teaching status (resident surgeon involvement), inpatient or outpatient status, and other case characteristics. $X$ is the average operating room time for the procedure. $Z$ is the number of standard deviations associated with the probability of having enough time to set up for the case, do the surgery, and clean up afterward. $S$ is the unbiased sample standard deviation of the operating room time for the procedure.

With $Z=0$, the probability is 0.50 for having sufficient time to set up for the case, do the procedure, and clean up afterwards; it is the same as using the average time as previously discussed. With $Z=1.645$, the probability is 0.95 for having sufficient time.

Unfortunately, many OR procedure time distributions display non-normal characteristics. Plots of OR procedure times usually reveal a truncation on the left side and a tail on the right side as shown in Fig. 1. This suggests that $O R$ procedure times might be better represented by a lognormal distribution. Because of this, predictions for individual cases could be improved if they were based on a data base constructed from the natural logs of the procedure time data. To predict the time to be scheduled for the procedure, Equation (2) should be used on the natural log data instead of Equation (1). This is the same as using Equation (1) on the transformed data and then taking the antilog of the result.

$$
P_{\mathrm{ij}} \cdots \mathrm{m}=e^{\left[\bar{X}_{\mathrm{ij}} \cdots \mathrm{m}+Z^{*} S_{\mathrm{ij}} \cdots \mathrm{m}\right]}
$$

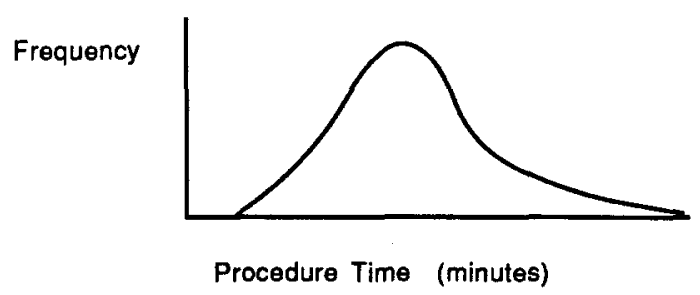

Figure 1. A plot of a typical procedure time. 
Equations (1) and (2) were presented for the purpose of determining the time required for a single case. If the scheduling policy of the OR suite is to assign fixed start times to cases throughout the day, then Equation (2) using a Log transformed data base should be used. An exception to this is when two or more cases on an "as follows" basis will be done. To determine the total amount of time that the room will be in use, Equation (3) should be used. Equation (3) should also be used to estimate the endpoint for the last case in a room (assuming that two or more procedures are to be performed) when the OR suite scheduling policy calls for all cases to be performed on an "as follows" basis. For this situation only the first case of the day in a room has a fixed starting time. Obviously, if only one case is to be performed in a room for the day, Equation (2) should be used regardless of the scheduling policy.

$$
T(N)=\sum_{h=1}^{N} \bar{X}_{\mathrm{ij}} \cdots \mathrm{m}+Z *\left(\sum_{h=1}^{N}\left(S_{\mathrm{ij}} \cdots \mathrm{m}\right)^{2}\right)^{1 / 2},
$$

where $T(N)$ is the total time needed for " $N$ " cases that are performed on an "as follows" basis.

As with Equation (1), Equation (3) also assumes that the cases' distributions are normally distributed. Unfortunately, the authors do not know of any equation that can be used with the transformed log data. Though some error will result, the size of this error will decrease as the number of cases $(\mathrm{N})$ increases. Summing the times derived with Equation (2) would significantly overestimate the amount of time required.

\section{DATA BASE REDUCTION METHODOLOGY}

In order to derive the greatest benefit from OR case time data, the data need to be subdivided into subsets that are not only clinically meaningful and consistent but also statistically similar for the purpose of predicting OR time needed for procedures. In addition, data subdivision needs to be done along lines that are conducive to the development of user-friendly microcomputer programs that can use the processed data for scheduling purposes.

Because OR time requirements are highly procedure dependent and because the data subsets need to be clinically meaningful, the authors have chosen the "set of codes" identifying the set of OR procedures (to be done during one trip to the OR) as the first factor to be used in data subdivision. Even though the time distributions for two different sets of procedures (e.g., one on the mouth and another on the foot) may be essentially the same, clinical meaningfulness of the subsets will be lost if the data are combined. Table 1 illustrates the type of summary statistics that one might see for data subdivided at the procedure set level for six different sets of procedures.

Further data analysis now can be attempted to refine the predictive power of the time estimates by subdividing the data by other factors, such as primary (staff) surgeon, case teaching status (resident involvement), patient inpatient/outpatient status, patient sex, patient age, etc. These particular factors are mentioned because they are patient and case attributes that should be known at the time the surgery is scheduled. Information not 
Table 1. A Partial Summary of Operating Room Time Data Subdivided at the Procedure Set Level

\begin{tabular}{lrrrrrrr}
\hline PC1 & PC2 & $\begin{array}{r}\text { AVG } \\
\text { TIME }\end{array}$ & $\begin{array}{l}\text { STDV } \\
\text { TIME }\end{array}$ & $\begin{array}{c}\text { AVG } \\
\text { LTIM }\end{array}$ & $\begin{array}{l}\text { STDV } \\
\text { LTIM }\end{array}$ & PRED & CNT \\
\hline 28.30 & 0.00 & 63.4 & 14.2 & 4.1266 & 0.2188 & 87.8 & 235 \\
47.00 & 0.00 & 77.6 & 19.0 & 4.3228 & 0.2431 & 112.5 & 61 \\
49.46 & 0.00 & 62.5 & 21.1 & 4.0980 & 0.2564 & 91.8 & 63 \\
53.00 & 0.00 & 71.8 & 16.1 & 4.2498 & 0.2174 & 100.2 & 241 \\
65.61 & 68.40 & 123.0 & 25.8 & 4.7906 & 0.2088 & 169.7 & 100 \\
79.35 & 0.00 & 143.3 & 32.8 & 4.9408 & 0.2191 & 200.6 & 128 \\
Total & & & & & & & 828 \\
\hline
\end{tabular}

The following is a key to the table's nomenclature:

28.30 Tonsillectomy with adenoidectomy

47.00 Appendectomy

49.46 Excision of hemorrhoids

53.00 Unilateral repair of inguinal hernia

65.61 Removal of both ovaries and tubes; same oper. episode

68.40 Total abdominal hysterectomy

79.35 Open reduction of fracture with internal fixation; femur

AVGTIME average room time (in minutes) for the procedure set

STDVTIME unbiased sample standard deviation (in minutes) for the distribution of the procedure set

AVGLTIM average of the natural logs of the procedure set room times

STDVLTIM unbiased sample standard deviation for the distribution of the natural logs of the procedure set room times

PRED predicted maximum length of time (in minutes) that the procedure set will require with a probability of 0.95 . It is derived from Equation (2). The specific equation is:

PRED $=e^{[\text {AVGLTIM }+(1.645 \times \text { STDVLTIM })]}$

CNT number of cases of data within the procedure set

known until after case scheduling, such as nursing/anesthesia personnel assignments and patient preoperative condition, cannot be used.

The objective of the methodology presented below is to determine the subsets of data within procedure sets that are statistically different from each other and that will produce better predictions of room time needed, based on the chosen factors and corresponding case attributes. Basically, the process involves subdividing the data into component subsets by the chosen factors and then pooling the subsets determined to be statistically similar in terms of their time requirements.

The methodology is designed to work with any size data set and with whatever factors an OR administrator may choose. For many types of procedures, however, sample size will limit the process of forming subsets of data beyond the procedure level. For other 
procedure sets, regardless of the amount of available data, subdivision will not be possible because a procedure and one or more specific factors may be incongruous, e.g., open heart surgery is not performed on an outpatient basis. Finally, attempted subdivision by multiple factors, though not necessarily incongruous in theory, may result in empty subsets due to OR policy, lack of data, and/or other reasons. To facilitate explanation, an example is presented based on procedure $\mathbf{5 3 . 0 0}$ (unilateral repair of inguinal hernia) data, summarized in Table 1. The factors selected as an example for data subdivision are primary surgeon and case teaching status (surgical resident involvement).

Before data subdivision is done for any procedure set, rules must be established to accommodate situations when potential subsets contain only single cases, i.e., insufficient data to compute variances. Though single case subsets simply could be excluded, it is desirable to combine them with one or more other single case subsets to maintain overall sample size. This complicates data management by having to maintain subset(s) in which one or more factors are disregarded in the formation of the initial component subsets. For our example, the following rules are used to handle single case subsets:

1. If a primary surgeon has performed only two cases with one being a teaching case and the other a nonteaching case, pool the two cases for the primary surgeon and disregard the teaching status factor.

2. If rule 1 fails, exclude the single case subset from analysis. It cannot be used in testing and pooling for this detailed set of factors, but it will be included in later analyses when, for example, teaching status is the only factor.

Table 2 illustrates the situation prior to final data subdivision with respect to the initial case count breakdown and other statistics. Because Surgeon 1 performed two cases, one teaching and one nonteaching, rule 1 will be applied when subdividing the data into their component subsets. Under rule 2, the single case performed by Surgeon 8 will be excluded from subset assignment for this round of testing and pooling. Table 3 shows these component subsets at the start of the testing and pooling process following subdivision, as well as the initial component subset identifiers (subset number) to be used to track the data during processing.

Table 2. Data Summary for Procedure 53.00 Subdivided by the Factors Primary Surgeon and Case Teaching Status

\begin{tabular}{rccccrr}
\hline $\begin{array}{l}\text { Primary } \\
\text { Surgeon }\end{array}$ & $\begin{array}{c}\text { Teaching } \\
\text { Status }\end{array}$ & $\begin{array}{c}\text { AVG } \\
\text { TIME }\end{array}$ & $\begin{array}{c}\text { STDV } \\
\text { TIME }\end{array}$ & $\begin{array}{c}\text { AVG } \\
\text { LTIM }\end{array}$ & $\begin{array}{r}\text { STDV } \\
\text { LTIM }\end{array}$ & CNT \\
\hline 1 & No & 45.0 & - & 3.8066 & - & 1 \\
1 & Yes & 75.0 & - & 4.3174 & - & 1 \\
2 & No & 50.0 & 0.0 & 3.9120 & 0.0000 & 3 \\
2 & Yes & 67.8 & 13.8 & 4.1964 & 0.2061 & 51 \\
3 & No & 64.0 & 10.7 & 4.1476 & 0.1680 & 5 \\
3 & Yes & 67.8 & 9.4 & 4.2073 & 0.1425 & 9 \\
4 & Yes & 67.3 & 11.8 & 4.1937 & 0.1750 & 66 \\
5 & No & 83.5 & 30.4 & 4.3905 & 0.3725 & 2 \\
5 & Yes & 90.3 & 15.1 & 4.4898 & 0.1659 & 18 \\
6 & No & 79.0 & 9.6 & 4.3638 & 0.1162 & 5 \\
6 & Yes & 77.8 & 13.5 & 4.3404 & 0.1605 & 37 \\
7 & Yes & 82.0 & 19.9 & 4.3809 & 0.2468 & 7 \\
8 & No & 55.0 & - & 4.0073 & - & 1 \\
\hline
\end{tabular}


Table 3. Data Summary for Procedure 53.00 Subdivided by the Factors of Primary Surgeon and Case Teaching Status

\begin{tabular}{ccccc}
\hline & \multicolumn{4}{c}{ Case Teaching Status } \\
\cline { 2 - 5 } & \multicolumn{2}{c}{ Teaching } & \multicolumn{2}{c}{ Nonteaching } \\
\cline { 2 - 5 } Primary & Subset & Case & Subset & Case \\
Surgeon & Number & Count & Number & Count \\
\hline 1 & $1^{a}$ & 1 & $1^{a}$ & 1 \\
2 & 3 & 51 & 2 & 3 \\
3 & 5 & 35 & 4 & 5 \\
4 & 7 & 66 & 6 & 9 \\
5 & 9 & 18 & 8 & 5 \\
6 & 11 & 37 & 10 & \\
7 & 12 & 7 & & 5 \\
\hline
\end{tabular}

${ }^{a}$ Pooled by Rule 1.

To determine if the data of two component subsets should be pooled, we must test the hypothesis that both subsets represent samples of data from the same population, i.e., the schedule time means and variances from Equation (4) for both subsets are equal. (An assumption is that if the variances are equal, the standards deviations are equal.) If equal, the hypothesis is accepted and the data are pooled (subsets combined); if either set of terms is not equal, then the hypothesis must be rejected. The first step is to test the variances $\left(S_{1}^{2}, S_{2}^{2}\right)$ using an equality of variance $F$ test. If no significant difference is found, then equality of the subsets' means $\left(X_{1}, X_{2}\right)$ is tested using a two-sample t-test. If the variances are not equal, there is no need to test the means.

In practice, testing and pooling occur in two separate stages. In Fig. 2, the decisions made during both stages of the process are graphically displayed for the procedure 53.00 example. On the far left side of the figure, the initial component subset identifiers from Table 3 are displayed. Though it is not possible to show all pair-wise tests in two dimensions, the asterisks $\left(^{*}\right)$ that fall in a vertical column indicate the existence of tests between one subset and every other subset in that column. Each vertical bar represents the combining of two sets and the pooling of their data. $P_{1}-P_{4}$ identify the aggregate subsets following stage one processing, and $A-F$ identify the final subset groups that are homogenous in terms of their subset means and standard deviations.

In the first stage of testing and pooling (see Fig. 3), processing is based only on the subsets' unbiased variances. One by one the subsets with the greatest similarity are combined and new comparisons are made between the pooled subset and the other remaining subsets. More specifically, processing begins by testing each pair of component subsets with the $F$ test for significant difference between their unbiased standard deviations (this requires $N(N-1) / 2$ tests for " $N$ " component subsets). Pooling then is done for only the two subsets with the largest significance level that is also greater than alpha (alpha $=0.05$ in the example). If no pair's significance level is greater than alpha, stage one processing is completed. If pooling does occur, then an unbiased overall standard deviation is computed for the pooled data of the combined subset, and pair-wise testing between the combined subset and the remaining subsets is done next. This process is repeated with subsequent pooling of subsets occurring each time the greatest significance 


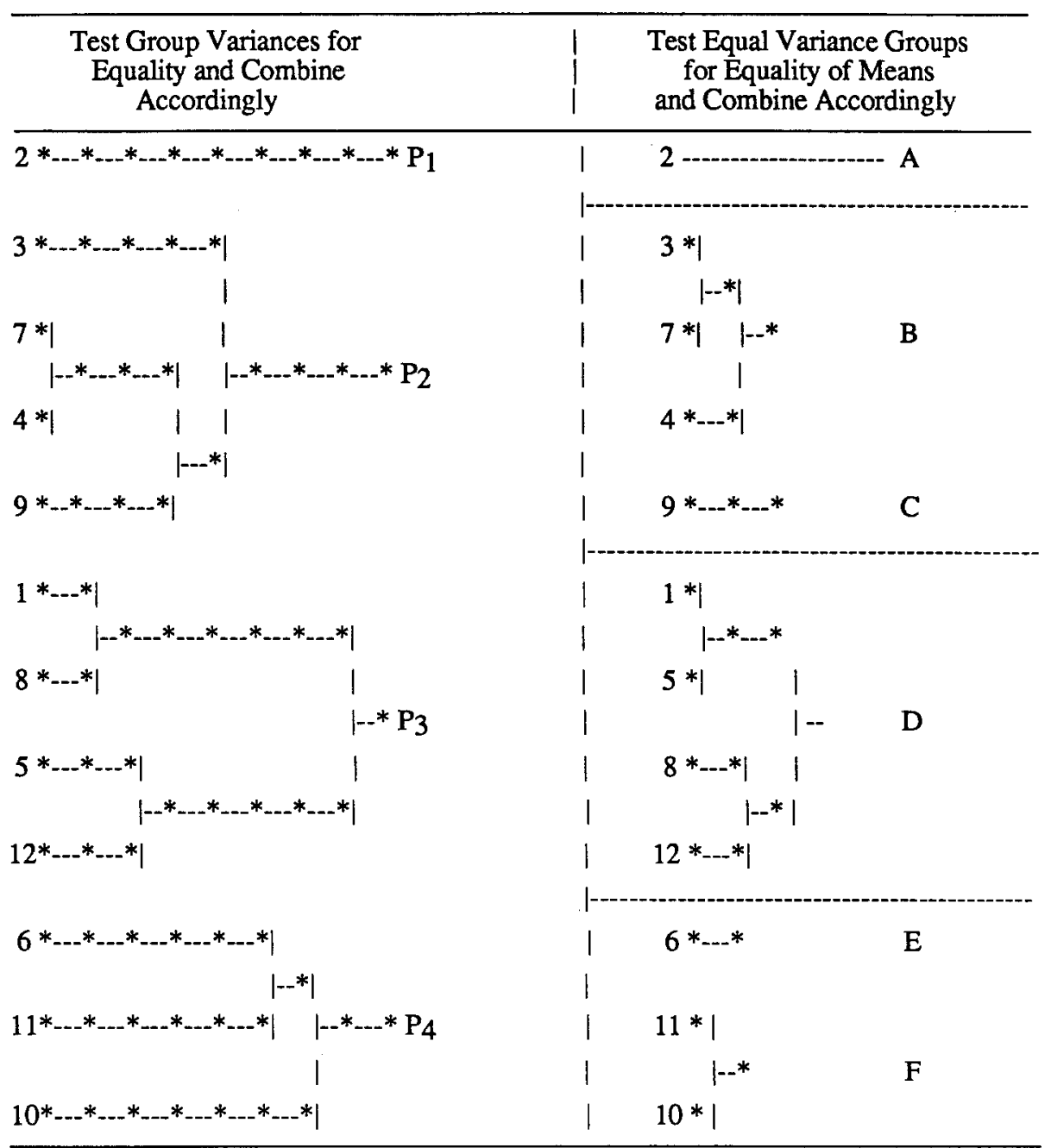

where:

-- The numbers identify original groups by surgeon and teaching status.

-- Asterisks "*" indicate comparisons with remaining groups.

-- Vertical bars indicate combining of subsets by pooling data.

-- P1 - P4 represent aggregate subsets with homogeous variances.

-- Capital letters in the far right column identify final groups.

Figure 2. A graphical display of the testing and pooling process performed on the component subsets of procedure 53.00 data.

level of a pair-wise test between the remaining pooled and unpooled subsets is greater than alpha. When stage one processing is completed, each resulting "aggregate" subset is homogeneous with respect to the variances of its component subset(s) and significantly different with respect to the component subset(s) of the other aggregates.

For the second stage, each aggregate is separated into its component subsets in 


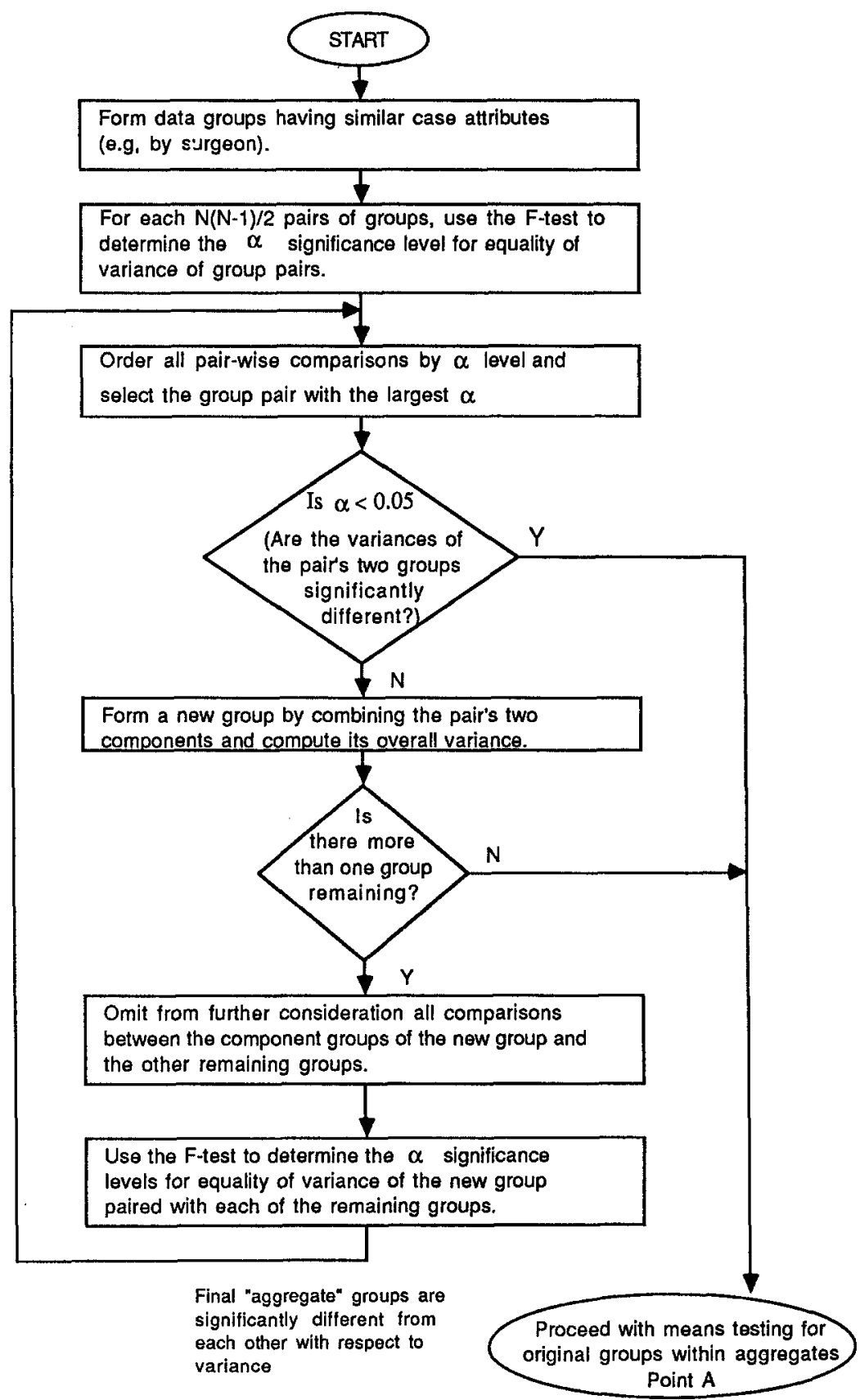

Figure 3. Flow chart for variance comparison.

testing and pooling of the latter based on the subsets' means (see Fig. 4). Processing is done on one aggregate at a time. This limits the scope of testing and pooling to only the component subsets from within the same aggregate which have no statistical differences in their standard deviations. Except for the use of $t$ tests instead of $F$ tests to assess 


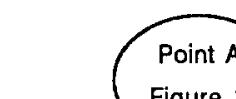

Figure 3

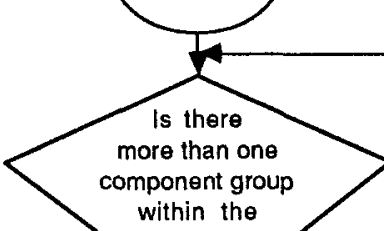

aggregrate?

$Y$

For each pair of groups, use the t-test to determine the $\alpha$

signiticance level for equality of means of the group pairs.

Order all pairwise comparisons by $\alpha$ level and select the

group pair with the largest $\alpha$.

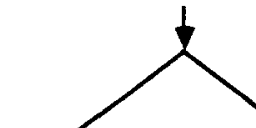

Is $\alpha<0.05$

(Are the means of

the pair's two groups

significantly

different?)

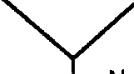

$\mathrm{N}$

Form a new group by combining the group pair's two components and compute the new group mean.

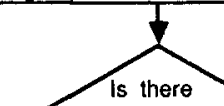

more than one

group

remaining?

$Y$

Omit from further consideration all comparisons between the component groups of the new group and the other remaining groups.

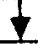

Use the t-test to determine the $\alpha$ significance levels for equality of means of the new group paired with each of the remaining groups.

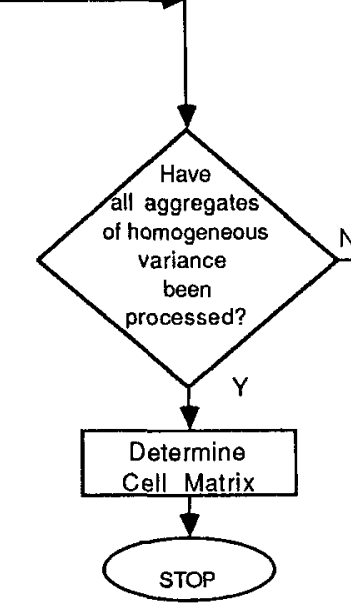

Figure 4. Flow chart for means comparisons. 
equality of the subsets' means, the algorithm for testing and pooling is essentially the same as in stage one.

For the procedure 53.00 example, Table 4 shows the statistics for both the intermediate $\left(P_{1} \ldots P_{4}\right)$ and final subsets $(\mathrm{A}, \mathrm{B} \ldots \mathrm{F})$ following testing and pooling. The headings in the table are as previously defined.

Based on the identifying component subset numbers, Table 5 depicts the final subset groups' assignments for scheduling purposes according to the primary surgeon and case teaching status factor. For example, operating room time for a case to be performed by Surgeon 3 on a nonteaching basis would be predicted by final subset group "B"'. Because the case for Surgeon 8 was excluded from testing and pooling, scheduling for Surgeon 8 and other surgeons not included in processing because of lack of cases cannot be determined from the analyses carried out so far.

Predictions for the time needs of Surgeon 8 and Surgeon 7, nonteaching will be minimal because they did one or fewer 53.00 procedures. However, there likely are to be instances when a time prediction will be needed for them, or when a new surgeon will want to do the procedure. For these instances, another round of testing and pooling will have to be done for all procedure 53.00 data factored only for case teaching status. This is accomplished using the same methodology as just described. Table 6 is an example of the result.

\section{THE SCHEDULING APPLICATION}

Once the data base has been analyzed, a system to predict times for scheduling purposes is necessary. Regardless of scheduling policy (fixed start time versus "as follows" scheduling), there always will be a need to compute the maximum times required for both single and multiple cases. Hence, the system must be capable of deriving time requirements based on both Equations (2) and (3). This implies that for each entry in the scheduling data base, there must be two sets of means and standard deviations, one

Table 4. Intermediate and Final Subset Information Derived from the Testing and Pooling of Procedure 53.00 Component Data Subsets Based on Primary Surgeon and Case Teaching Status

\begin{tabular}{ccrccrr}
\hline & AVG & STD & AVG & STD & & \\
DATA SET & TIME & TIME & LTIM & LTIM & PRED & CNT \\
\hline $\mathrm{P}_{1}$ & 50.0 & 0.0 & 3.9120 & 0.0000 & 50.0 & 3 \\
$\mathrm{P}_{2}$ & 70.3 & 15.0 & 4.2311 & 0.2093 & 97.1 & 140 \\
$\mathrm{P}_{3}$ & 73.2 & 20.8 & 4.2568 & 0.2699 & 110.0 & 46 \\
$\mathrm{P}_{4}$ & 76.1 & 13.0 & 4.3192 & 0.1602 & 97.8 & 51 \\
$\mathrm{~A}$ & 50.0 & 0.0 & 3.9120 & 0.0000 & 50.0 & 3 \\
$\mathrm{~B}$ & 67.4 & 12.6 & 4.1929 & 0.1871 & 90.1 & 122 \\
$\mathrm{C}$ & 90.3 & 15.1 & 4.4898 & 0.1659 & 117.1 & 18 \\
D & 73.2 & 20.8 & 4.2568 & 0.2699 & 110.0 & 46 \\
$\mathrm{E}$ & 67.8 & 9.4 & 4.2073 & 0.1425 & 84.9 & 9 \\
$\mathrm{~F}$ & 77.9 & 13.0 & 4.3432 & 0.1549 & 99.3 & 42 \\
\hline
\end{tabular}


Table 5. Final Subset Group Assignments

\begin{tabular}{ccc}
\hline $\begin{array}{c}\text { Primary } \\
\text { Surgeon }\end{array}$ & $\begin{array}{c}\text { Teaching } \\
\text { Case }\end{array}$ & $\begin{array}{c}\text { Nonteaching } \\
\text { Case }\end{array}$ \\
\hline 1 & D & D \\
2 & B & A \\
3 & D & B \\
4 & B & E \\
5 & C & D \\
6 & F & F \\
7 & D & $* a$ \\
8 & $* a$ & $* a$ \\
\hline
\end{tabular}

${ }^{a}$ No group assignment because of rule 2 .

representing $\log$ data and one representing the untransformed data. An alternative arrangement would be to have the means and standard deviations of the untransformed data along with precalculated estimates for single procedure set scheduling times. This, however, would restrict time estimates for single procedure sets to values based on a predetermined value of $Z$ (as in Table 4).

To deliver the greatest benefit, the system must render scheduling time estimates for as many types of procedures as possible. Because of sample size limitations, there will continue to be a need for estimates from surgeons and/or OR schedulers for infrequently performed procedures and for surgeons doing a particular type of procedure for the first time. The system should produce some time estimate even if it has no specific data for one or more of the factors used in the full-scale factor analysis. Therefore, the system's data base will contain a degree of redundancy to enable the scheduler to use the most appropriate set of data for the circumstances.

For example, suppose that testing and pooling for only the teaching status factor produced the results shown in Table 6 . Table 7 then would provide the type of scheduling data base entries for procedure 53.00 based on both multiple factor processing (primary surgeon and teaching status) and single factor processing (teaching status).

In practice, the system first would prompt the user to enter information on all factors used for testing and pooling, i.e., procedure codes, primary surgeon codes, and the expected case teaching status. Next, the system would locate hierarchically the data base record containing the statistics of the subset that most closely matched the factors entered by the user. In Table 7 records 786 through 798 contain the statistics for all surgeons for which surgeon-specific predictions can be made, whereas records 784 and 785 contain the statistics for all other surgeons depending on the teaching status of the case to be scheduled.

Table 6. Hypothetical Final Subset Information from Testing and Pooling of Procedure 53.00 Component Data Subsets Subdivided by Only Case Teaching Status

\begin{tabular}{lccccrr}
\hline & AVG & STD & AVG & STD & & \\
DATA SET & TIME & TIME & LTIM & LTIM & PRED & CNT \\
\hline Teaching & 73.4 & 16.2 & 4.2778 & 0.2172 & 103.0 & 215 \\
Nonteaching & 67.0 & 14.5 & 4.1828 & 0.2119 & 92.9 & 26 \\
\hline
\end{tabular}


Table 7. Example of Scheduling System Data Base for Procedure 53.00 Based on Information from Table 4 and Table 6

\begin{tabular}{|c|c|c|c|c|c|c|c|c|}
\hline \multicolumn{5}{|c|}{ Data Base Factors } & \multicolumn{4}{|c|}{ Final Subset Group Statistics } \\
\hline $\begin{array}{l}\text { Record } \\
\text { Number }\end{array}$ & PCl & $\mathrm{PC} 2$ & $\begin{array}{l}\text { Surgeon } \\
\left(_{(i)^{a}}\right.\end{array}$ & $\begin{array}{c}\text { Teach } \\
\left(j^{b}\right)^{b}\end{array}$ & $\begin{array}{l}\text { AVG } \\
\text { TIME }\end{array}$ & $\begin{array}{l}\text { STDV } \\
\text { TIME }\end{array}$ & $\begin{array}{c}\text { AVG } \\
\text { LTIM }\end{array}$ & $\begin{array}{l}\text { STDV } \\
\text { LTIM }\end{array}$ \\
\hline 784 & 53.00 & 0.00 & 0 & 1 & 73.4 & 16.2 & 4.2778 & 0.2172 \\
\hline 785 & 53.00 & 0.00 & 0 & 2 & 67.0 & 14.5 & 4.1828 & 0.2119 \\
\hline 786 & 53.00 & 0.00 & 1 & 1 & 73.2 & 20.8 & 4.2568 & 0.2699 \\
\hline 787 & 53.00 & 0.00 & 1 & 2 & 73.2 & 20.8 & 4.2568 & 0.2699 \\
\hline 788 & 53.00 & 0.00 & 2 & 1 & 67.4 & 12.6 & 4.1929 & 0.1871 \\
\hline 789 & 53.00 & 0.00 & 2 & 2 & 50.0 & 0.0 & 3.9120 & 0.0000 \\
\hline 790 & 53.00 & 0.00 & 3 & 1 & 73.2 & 20.8 & 4.2568 & 0.2699 \\
\hline 791 & 53.00 & 0.00 & 3 & 2 & 67.4 & 12.6 & 4.1929 & 0.1871 \\
\hline 792 & 53.00 & 0.00 & 4 & 1 & 67.4 & 12.6 & 4.1929 & 0.1871 \\
\hline 793 & 53.00 & 0.00 & 4 & 2 & 67.8 & 9.4 & 4.2073 & 0.1425 \\
\hline 794 & 53.00 & 0.00 & 5 & 1 & 90.3 & 15.1 & 4.4898 & 0.1659 \\
\hline 795 & 53.00 & 0.00 & 5 & 2 & 73.2 & 20.8 & 4.2568 & 0.2699 \\
\hline 796 & 53.00 & 0.00 & 6 & 1 & 77.9 & 13.0 & 4.3432 & 0.1549 \\
\hline 797 & 53.00 & 0.00 & 6 & 2 & 77.9 & 13.0 & 4.3432 & 0.1549 \\
\hline 798 & 53.00 & 0.00 & 7 & 1 & 73.2 & 20.8 & 4.2568 & 0.2699 \\
\hline
\end{tabular}

"A value of " 0 " for Surgeon indicates that the factor was not used in forming the subset.

${ }^{b}$ In the Teach column, " 1 " indicates a teaching case and " 2 "' indicates a nonteaching case.

Application of the scheduling system data base would depend on the type of scheduling function to be performed. For each case to be scheduled, the computer would uniquely identify the appropriate data base record to be used by the scheduler's inputs. If only a single case were to be scheduled, the estimated time required would be computed from the values in the last two columns and Equation (2). If an "as follows" scheduling function were to be processed, values from the first two columns for each of the "as follows" cases and Equation (3) would be used to compute the overall time required.

\section{MATRIX MAINTENANCE}

In practice, the methodology presented herein would be incorporated into a computer program that would be run every three months to produce a matrix like Table 7 . As the data base increased in size, the methodology would produce more precise schedule times because of the increased power of the statistical tests. Consideration also must be given to purging the data base of particular procedure times if the following conditions were found to exist:

1. If the method of doing a particular procedure changed.

2. If a surgeon left the staff.

3. If errors occurred concerning the elapsed time of a procedure. This typically occurs when there are delays in doing a procedure that are not excluded prior to posting to the data base.

4. Changes in the methods in determining the start and stop times of doing a procedure. 


\section{CONCLUSION}

A methodology with examples has been presented to produce an OR procedure historical data base to provide schedule times for future procedures. Use of the methodology requires a microcomputer because of the computations necessary to perform the tests, the $\log$ transforms and the repooling of data. The result is schedule times that are established according to hospital policy with the specified probability of having enough time to do the procedures. These schedule times, when properly integrated into the OR scheduling procedure, should greatly reduce the administrative problems of late starts, poor utilization, and excessive overtime.

\section{REFERENCES}

1. Hancock, W.M., and Walter, P.F., The ASCS Inpatient Admission and Scheduling and Control System, Health Administration Press, Ann Arbor, Mich., Chapter IX, 1984.

2. Hancock, W.M., and Walter, P.F., Reduce hospital costs with admissions and operating scheduling systems. Software Healthcare 42-46, 1986.

3. Neter, J., Wasserman, W., and Kutner, M.H., Applied Linear Statistical Models, Richard P. Irwin, Inc., 2nd Edition, 1985.

4. Montgomery, D.C., Design and Analysis of Experiments, Wiley (second edition), 1984. 